\title{
T-shaped and H-shaped polymers constructed from UV-induced strain promoted azide-alkyne cycloaddition reaction
}

\author{
Wen Zhu ${ }^{\text {a }}, \mathrm{Zi} \mathrm{Li}^{\text {a }}$, Peng Sun ${ }^{\mathrm{a}}$, Lixia Ren ${ }^{\mathrm{b}}$, Ke Zhang ${ }^{\mathrm{a}, *}$ \\ ${ }^{\text {a }}$ Laboratory of Polymer Physics and Chemistry, Institute of Chemistry, The Chinese Academy of \\ Sciences, Beijing 100190, China \\ ${ }^{\mathrm{b}}$ School of Materials Science and Engineering, Tianjin University, No. 92 Weijin Road, Tianjin 300072, \\ China \\ *Corresponding author: K. Zhang (E-mail: kzhang@iccas.ac.cn)
}

\begin{abstract}
Topological polymers with $\mathrm{T}$-shaped and $\mathrm{H}$-shaped molecular architecture were built on the combination of atom transfer radical polymerization (ATRP) and UV-induced strain promoted azide-alkyne cycloaddition (SPAAC) reaction. In the presence of a cyclopropenone-masked dibenzocyclooctyne functionalized dibromo ATRP initiator, reactive polystyrene (PS) was synthesized to have a cyclopropenone-masked dibenzocyclooctyne group in the middle of polymer chain. After releasing dibenzocyclooctyne from deprotecting cyclopropenone-masked dibenzocyclooctyne under UV irradiation, T-shaped and $\mathrm{H}$-shaped topological polymers were constructed by reacting PS with mono-end and di-end azide functionalized poly(ethylene oxide) (PEO), respectively, based on the SPAAC reaction.
\end{abstract}


Keywords: Atom transfer radical polymerization; H-shaped polymer; T-shaped polymer; Topological polymer; UV-induced strain promoted azide-alkyne cycloaddition

\section{Introduction}

Polymer topology plays an important role in determining the properties and functionalities of polymer materials. ${ }^{1}$ The synthesis of topological polymers has been always a core direction in the field of polymer chemistry. Thanks to the development of modern living/quasiliving polymerization ${ }^{2}$ techniques (LP) and the wide application of click chemistry in polymer science, a variety of polymer topology has been fabricated based on their combination including starlike, ${ }^{3}$ brushlike, ${ }^{4}$ grafted,${ }^{5}$ hyperbranched, ${ }^{6}$ cyclic macromolecular ${ }^{7}$ architectures and polymer networks. ${ }^{8}$ In this strategy, LP was used to prepare varied polymer building blocks functionalized with clickable groups. The click reactions were then used to couple the related building blocks to form the topological polymers. As the simplest branched polymers, synthesis of T-shaped (3-arm star) and $\mathrm{H}$-shaped polymers could be viewed as the golden candidate to evaluate the combination of LP and click chemistry for fabricating polymer topology.

To date, a series of LP and click chemistry combined methods have been developed for the formation of T-shaped and H-shaped polymers. Based on the LP, a series of click reactions have been used to fabricate the T-shaped and H-shaped polymers, including copper-catalyzed azide-alkyne cycloaddition $(\mathrm{CuAAC}),{ }^{3,9-13}$ Diels-Alder reaction, ${ }^{14,15}$ and thiol-ene reaction. ${ }^{16,17}$ In these techniques, CuAAC was one of most important click reactions due to its high efficiency and small sized reaction groups. In addition, the azide group could be easily introduced at the end of polymers based on atom transfer radical polymerization (ATRP) and substitution of bromide end group by azide. To achieve high 
reaction efficiency, however, a large amount of copper catalysts was required by CuAAC. This may cause the contamination of the resultant topological polymers and restrict their application in the field of advanced technologies such as biology and photoelectronics.

Recently, the strain promoted azide-alkyne cycloaddition (SPAAC) reaction was developed as a bio-orthogonal reaction to circumvent the awkward situation of CuAAC in biology. ${ }^{18}$ By confining an alkyne in a small ring molecule, the strain activated alkyne group could efficiently react with azide group at room temperature requiring no any catalyst. Since its birth, SPAAC has been widely used in the formation of bio-conjugates in vitro and in vivo. ${ }^{19}$ In addition, SPAAC has also been applied to prepare polymeric materials including polymer surface brush ${ }^{20}$ and networks. ${ }^{21}$ Considering the importance of SPAAC click reaction, we synthesized a dibenzocyclooctyne functionalized ATRP initiator and systematically studied its ATRP behavior. ${ }^{22}$ For polymerizing styrenic and methacrylate monomers, the polymerization held living characteristics. For polymerizing acrylate monomers, however, the polymerization lost living behavior. Based on the combination of ATRP and SPAAC, we successfully prepared the block, star and brush topological polymers. ${ }^{22,23}$ Furthermore, by designing the ATRP initiator containing a cyclopropenone-masked dibenzocyclooctyne, the well-defined polystyrenics, polymethacrylates and polyacrylates could be produced by a standard ATRP protocol and the polymerizations all behaved living characteristics. ${ }^{24}$ Under UV irradiation, cyclopropenone-masked dibenzocyclooctyne quantitatively released the reactive dibenzocyclooctyne group, which in-situ reacted with azide by the SPAAC mechanism. By the combinations of ATRP/UV-induced SPAAC ${ }^{25}$ and macromolecular design via the interchange of xanthate (MADIX)/UV-induced SPAAC,${ }^{26}$ the cyclic polymer topology has been successfully prepared. 
As a continuous contribution in this research topic herein, $\mathrm{T}$-shaped and $\mathrm{H}$-shaped polymers were prepared by the combination of ATRP and UV-induced SPAAC click reaction. As shown in Scheme 1, by a difunctional ATRP initiator, the well-defined linear PS building blocks were synthesized from ATRP to have a cyclopropenone-masked dibenzocyclooctyne in the middle of the polymer chain. After quantitatively releasing dibenzocyclooctyne group under UV irradiation, T-shaped and H-shaped hybrid copolymers were prepared by reacting linear PS building blocks with mono- and di-end azide functionalized poly(ethylene oxide) (PEO), respectively. 


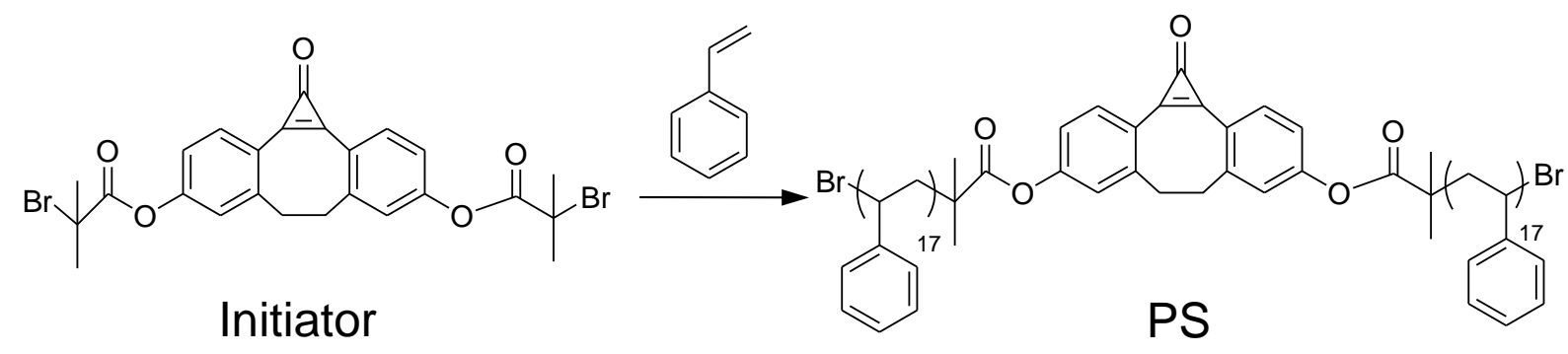

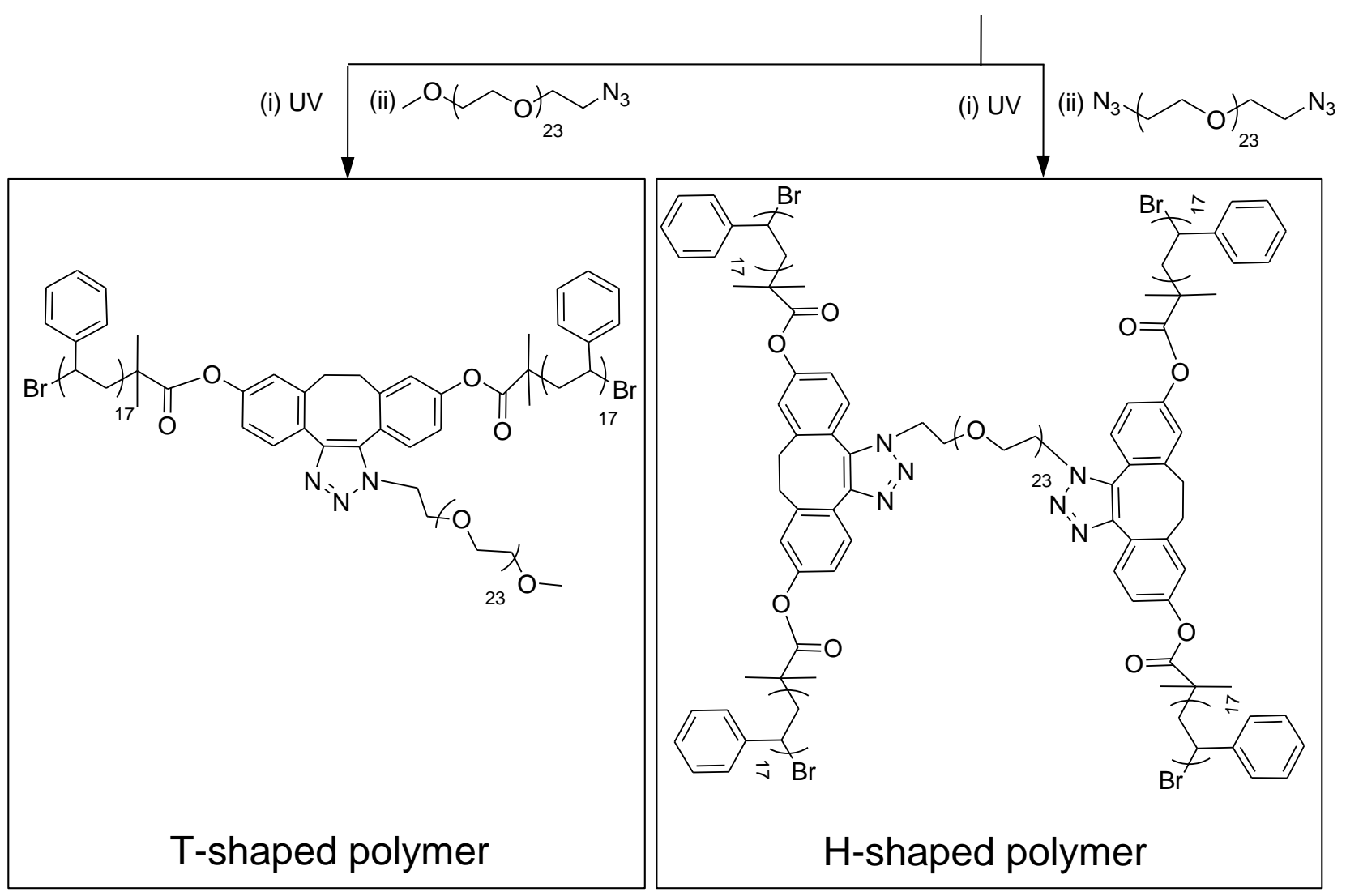

Scheme 1. Synthetic route of T-shaped and H-shaped polymers.

\section{Experimental section}

\subsection{Materials}

Styrene (St, 99\%, Aldrich) was dried over $\mathrm{CaH}_{2}$ and distilled under reduced pressure. Copper (I) bromide $(\mathrm{CuBr}, 98 \%$, J\&K) was washed by acetic acid and ethanol. Tetrahydrofuran (THF, AR, Beijing Chemical Reagent Co.) was distilled from sodium/benzophenone. Cyclopropenone-masked 
dibenzocyclooctyne functionalized ATRP initiator, ${ }^{24}$ mono- and di-end azide functionalized PEO $^{22}$ $\left(\mathrm{PEO}_{23}-\mathrm{N}_{3}\right.$ and $\left.\mathrm{N}_{3}-\mathrm{PEO}_{23}-\mathrm{N}_{3}\right)$ were synthesized according to the literatures. Pentamethyldiethylenetriamine (PMDETA, 99\%, Energy Chemical), anisole (AR, Beijing Chemical Reagent Co.) and other reagents were purchased from commercial retailers and used as received. A low pressure mercury lamp (120 W) (CEL-LPH120-254, Beijing China Education Au-light co.Ltd) was used as the UV light source.

\subsection{Measurements}

${ }^{1} \mathrm{H}$ NMR spectra were obtained on a Bruker DMX400 spectrometer. $\mathrm{CDCl}_{3}$ was used as solvent and TMS as internal standard. Apparent molecular weights $\left(M_{\mathrm{n}, \mathrm{GPC}}\right)$ and molecular weight distributions $\left(M_{\mathrm{w}} / M_{\mathrm{n}}\right)$ of the polymers were determined by gel permeation chromatography (GPC) equipped with a Waters 1515 isocratic HPLC pump, a Waters 2414 refractive index detector, and a combination of column Styragel HT-2, HT-3, HT-4 and HT-5. Linear polystyrene standards were applied for calibration. The eluent was THF at a flow rate of $1 \mathrm{~mL} / \mathrm{min}$ at $35^{\circ} \mathrm{C}$. The preparative GPC (SHIMADZU LC-20AR) equipped with a LC-20AR pump, a RID-20A refractive index detector, and an Agilent Plgel Mixed-D column. The eluent was THF at a flow rate of $10 \mathrm{~mL} / \mathrm{min}$ at room temperature. UV spectra were recorded using a TU-1901 UV-Visible Spectrophotometer. IR spectra were recorded using a Nicolet AVATAR 330 FT-IR Spectrophotometer.

\subsection{Synthesis of linear PS}

St (1.56 g, $15 \mathrm{mmol})$, ATRP initiator (28 mg, $0.05 \mathrm{mmol})$, PMDETA (9 mg, $0.05 \mathrm{mmol})$ and anisole (1.5 $\mathrm{g}$ as a solvent) were mixed into a $25 \mathrm{~mL}$ Schlenk tube. After degassing through three freeze-pump-thaw cycles, $\mathrm{CuBr}(7.2 \mathrm{mg}, 0.05 \mathrm{mmol})$ was added to the mixture in frozen state under $\mathrm{N}_{2}$. 
After another three freeze-pump-thaw cycles, the reaction was stirred at $80{ }^{\circ} \mathrm{C}$ for $40 \mathrm{~min}$, and then quenched by exposing to air. The mixture was diluted with $150 \mathrm{~mL} \mathrm{CH}_{2} \mathrm{Cl}_{2}$ and passed through a short basic alumina column to remove the catalyst. The solution was concentrated and precipitated three times in methanol. The polymer was dried under vacuum.

\subsection{Synthesis of T-shaped polymer}

Linear PS (40 mg, $0.01 \mathrm{mmol}$ ) in $100 \mathrm{~mL}$ THF was stirred under UV irradiation for $3 \mathrm{~h}$ at room temperature. The solution was then concentrated to $2 \mathrm{~mL}$. After adding $\mathrm{PEO}_{23}-\mathrm{N}_{3}(20 \mathrm{mg}, 0.02 \mathrm{mmol})$ in situ, the mixture was stirred at room temperature in air for another $12 \mathrm{~h}$. The solution was precipitated in methanol to remove excess $\mathrm{PEO}_{23}-\mathrm{N}_{3}$. The polymer was dried under vacuum.

\subsection{Synthesis of $\mathbf{H}$-shaped polymer}

Linear PS (80 mg, $0.02 \mathrm{mmol}$ ) in $200 \mathrm{~mL}$ THF was stirred under UV irradiation for $3 \mathrm{~h}$ at room temperature. The solution was then concentrated to $2 \mathrm{~mL}$. After adding $\mathrm{N}_{3}-\mathrm{PEO}_{23}-\mathrm{N}_{3}(5 \mathrm{mg}, 0.005 \mathrm{mmol})$ in situ, the mixture was stirred at room temperature in air for another $36 \mathrm{~h}$. The product $(85 \mathrm{mg}, 42.5$ $\mathrm{mg} / \mathrm{mL}$ in THF) was purified directly by GPC fractionation to remove excess PS. The polymer was dried under vacuum.

\section{Results and discussion}

\subsection{Synthesis and characterization of linear PS}

Cyclopropenone-masked dibenzocyclooctyne functionalized PS was synthesized via ATRP, using the

functional ATRP initiator. Fig. S1 (Supporting Information) shows the synthetic route of cyclopropenone-masked dibenzocyclooctyne functionalized ATRP initiator, and the experimental details 
were previously reported. ${ }^{24}$ The ${ }^{1} \mathrm{H}$ NMR spectrum and the related peak assignments are shown in Fig. S2, which clearly indicated the successful preparation of the initiator. Then it was applied to synthesize PS via ATRP. Using a polymerization condition of $[\mathrm{St}]_{0}:[\text { initiator }]_{0}:[\mathrm{CuBr}]_{0}:[\mathrm{PMDETA}]_{0}=300: 1: 1: 1$ and $[\mathrm{St}]_{0}=10 \mathrm{~mol} / \mathrm{L}$ in anisole, the linear PS was obtained after $40 \mathrm{~min}$ polymerization at $80{ }^{\circ} \mathrm{C}$. Fig. S3 shows the ${ }^{1} \mathrm{H}$ NMR spectrum of the raw polymerization solution without purification, in which the signals of $\mathrm{H}_{\mathrm{a} 1}$ and $\left(\mathrm{H}_{\mathrm{a} 2}+\mathrm{H}_{\mathrm{b} 2}\right)$ were assigned to the protons of St monomer $(2 \mathrm{H})$ and the resultant PS $(3 \mathrm{H})$ respectively. By virtue of the ratio of $\mathrm{Area}_{(\mathrm{Ha} 2+\mathrm{Hb} 2)} /\left(\frac{3}{2} \mathrm{Area} \mathrm{Ha}_{1}+\mathrm{Area}_{(\mathrm{Ha} 2+\mathrm{Hb} 2)}\right)$, the St conversion was calculated as $11.2 \%$. This produced a degree of polymerization (DP) of 34 for the resultant cyclopropenone-masked dibenzocyclooctyne functionalized PS. The ${ }^{1} \mathrm{H}$ NMR spectrum of purified linear PS is shown in Fig. 1 including the peak assignments. The characteristic signals of $\mathrm{H}_{\mathrm{a}}, \mathrm{H}_{\mathrm{d}}$ and $\mathrm{H}_{\mathrm{d}}$ ' were preserved in PS from the ATRP initiator. The signals of $\mathrm{H}_{b}$ and $\mathrm{H}_{c}$ of the initiator were overlapped with the $\mathrm{H}$ proton signals of phenyl groups in PS. Peak $\mathrm{H}_{\mathrm{k}}(2 \mathrm{H})$ at $4.41 \mathrm{ppm}$ represented the $\mathrm{H}$ of terminal St unit close to Br. A peak area ratio of 1 was obtained between $\mathrm{H}_{a}(2 \mathrm{H})$ and $\mathrm{H}_{d}(2 \mathrm{H})$, which indicated that the cyclopropenone-masked dibenzocyclooctyne group was successfully introduced into each PS chain. In addition, the peak area ratio between $\mathrm{H}_{h}(10 \mathrm{nH})$ and $\mathrm{H}_{\mathrm{k}}(2 \mathrm{H})$ was 87 , and DP was further calculated as 34 , which was consistent with that calculated by monomer conversion. Thus the $M_{\mathrm{n}, \mathrm{NMR}}$ of PS was 4100. 


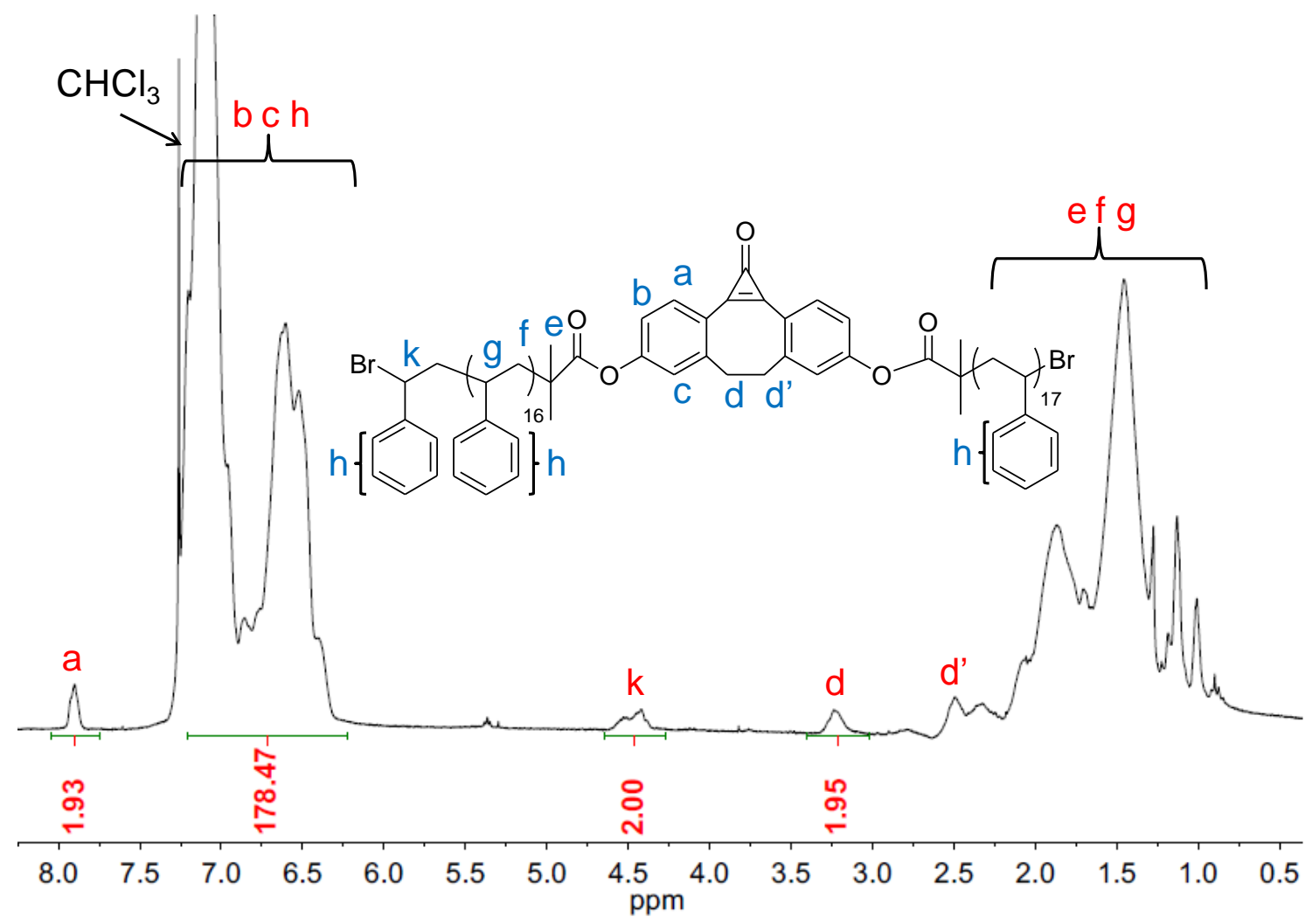

Fig. 1. ${ }^{1} \mathrm{H}$ NMR spectrum of cyclopropenone-masked dibenzocyclooctyne functionalized $\mathrm{PS}$ in $\mathrm{CDCl}_{3}$.

Fig. 2A (black) shows the GPC curve of linear PS, in which a well-defined monomodal and symmetric peak was observed. The $M_{\mathrm{n}, \mathrm{GPC}}$ and $M_{\mathrm{w}} M_{\mathrm{n}}$ were integrated as 4880 and 1.04 , respectively. It is reported that cyclopropenone-masked dibenzocyclooctyne ATRP initiator possessed a characteristic peak at $314 \mathrm{~nm}$ in the UV-Vis spectrum. ${ }^{24}$ As shown in Fig. 3A (black), linear PS inherited the peak of $314 \mathrm{~nm}$ from the ATRP initiator. These results clearly indicated the successful preparation of linear PS with the cyclopropenone-masked dibenzocyclooctyne group in the middle of polymer chain. 

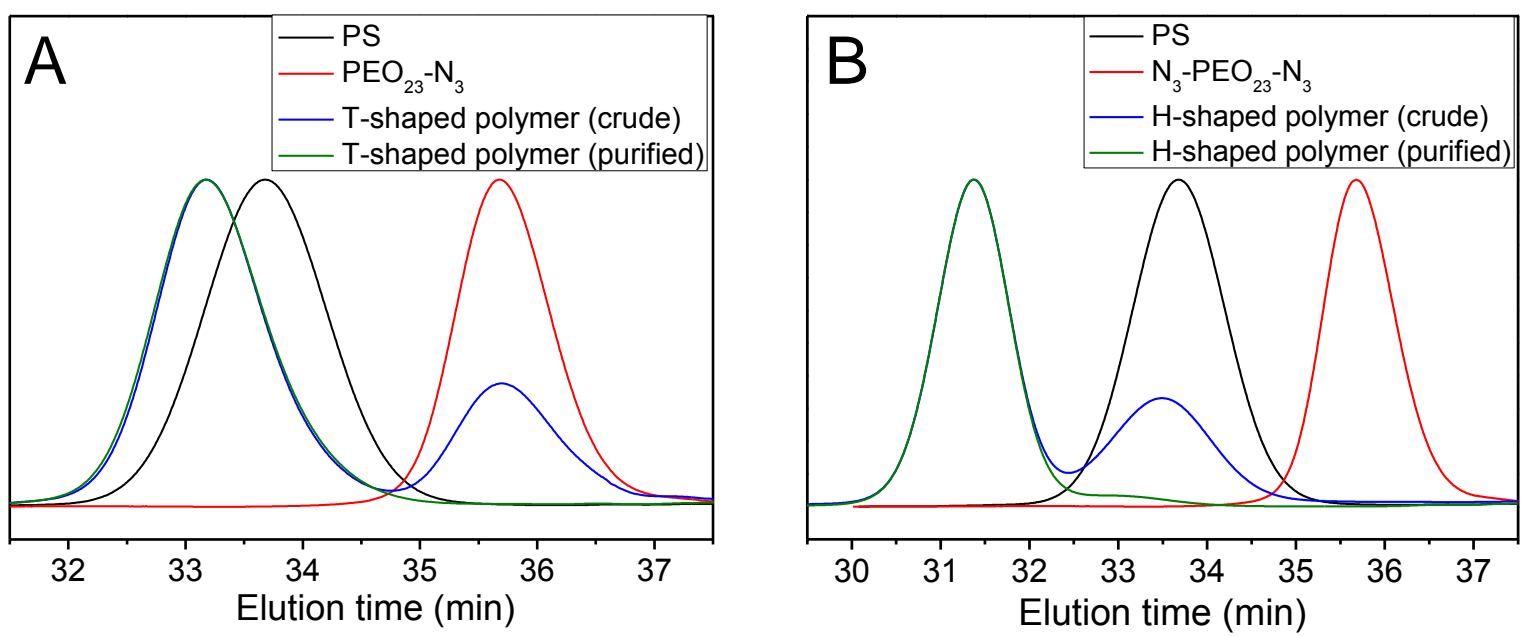

Fig. 2. (A) GPC curves of PS (black), $\mathrm{PEO}_{23}-\mathrm{N}_{3}$ (red), crude (blue) and purified (olive) $\mathrm{T}$-shaped polymers. (B) GPC curves of PS (black), $\mathrm{N}_{3}-\mathrm{PEO}_{23}-\mathrm{N}_{3}$ (red), crude (blue) and purified (olive) H-shaped polymers. THF was used as the eluent and polystyrene standards were used for the calibration.
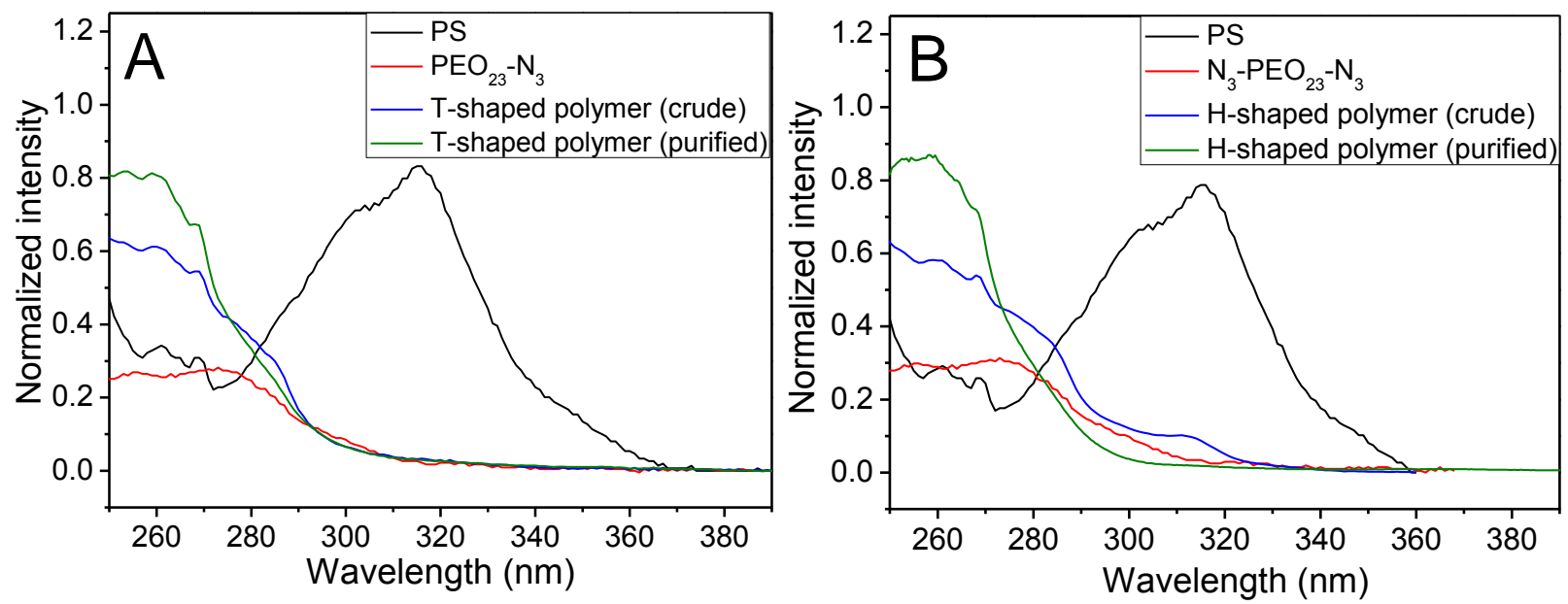

Fig. 3. (A) UV-Vis spectra of PS (black), $\mathrm{PEO}_{23}-\mathrm{N}_{3}$ (red), crude (blue) and purified (olive) T-shaped polymers in THF. (B) UV-Vis spectra of PS (black), $\mathrm{N}_{3}-\mathrm{PEO}_{23}-\mathrm{N}_{3}$ (red), crude (blue) and purified (olive) H-shaped polymers in THF.

\subsection{Synthesis and characterization of T-shaped polymer}


The cyclopropenone-masked dibenzocyclooctyne functionalized PS and $\mathrm{PEO}_{23}-\mathrm{N}_{3}$ were selected to prepare T-shaped polymers via the UV-induced SPAAC. $\mathrm{PEO}_{23}-\mathrm{N}_{3}$ was synthesized according to the route of Fig. S4, and the details were previously reported. ${ }^{22}{ }^{1} \mathrm{H}$ NMR spectrum of $\mathrm{PEO}_{23}-\mathrm{N}_{3}$ is shown in Fig. S5, and the peak area of $\mathrm{H}_{\mathrm{a}}(94 \mathrm{H})$ and $\mathrm{H}_{\mathrm{b}}(2 \mathrm{H})$ was 47.25 (theoretical value is 47 ), indicating the hydroxyl end group has completely transformed to azide group. The $M_{\mathrm{n}, \mathrm{NMR}}$ of $\mathrm{PEO}_{23}-\mathrm{N}_{3}$ was 1100 . Fig. $4 \mathrm{~A}$ (red) shows the IR spectrum of $\mathrm{PEO}_{23}-\mathrm{N}_{3}$, in which the peak at $2100 \mathrm{~cm}^{-1}$ was ascribed to azide group. Fig. 2A (red) shows the GPC curve of $\mathrm{PEO}_{23}-\mathrm{N}_{3}$, and the $M_{\mathrm{n}, \mathrm{GPC}}$ and $M_{\mathrm{w}} / M_{\mathrm{n}}$ were integrated as 2140 and 1.03 , respectively.

A low pressure mercury lamp was used as the UV light source to deprotect the cyclopropenone-masked dibenzocyclooctyne group. A THF dilute solution $\left(1 \times 10^{-4} \mathrm{~mol} / \mathrm{L}\right)$ of PS was irradiated by UV light for $3 \mathrm{~h}$. Then the solution was concentrated to $2 \mathrm{~mL}$ and 2 equivalent of $\mathrm{PEO}_{23}-\mathrm{N}_{3}$ was added in situ. After $12 \mathrm{~h}$ reaction at room temperature in air, T-shaped PS-PEO copolymer was conveniently obtained. The crude product was characterized by GPC, UV-Vis and IR. As shown in Fig. 2A (blue), the GPC curve of the newly formed polymer moved to a higher molecular weight direction, but remained the mono-modal and symmetric peak shape compared to that of PS (black). Reasonably, there was a surplus of excess $\mathrm{PEO}_{23}-\mathrm{N}_{3}$ in the GPC curve. This clearly indicated the successful preparation of the T-shaped polymer via the UV-induced SPAAC reaction. In the UV-Vis spectrum of Fig. 3A (blue), the absorption peak at $314 \mathrm{~nm}$ was disappeared completely, indicating the complete consumption of the cyclopropenone-masked dibenzocyclooctyne groups in linear PS.

The crude product was purified by precipitating in methanol to remove excess $\mathrm{PEO}_{23}-\mathrm{N}_{3}$. GPC curve of the purified T-shaped polymer shows no residual $\mathrm{PEO}_{23}-\mathrm{N}_{3}$, and the $M_{\mathrm{n}, \mathrm{GPC}}$ and $M_{\mathrm{w}} / M_{\mathrm{n}}$ were 
integrated as 5620 and 1.03, respectively (Fig. 2A, olive). The IR spectrum of the purified T-shaped polymer (Fig. 4A, olive) shows no vibration peak at $2100 \mathrm{~cm}^{-1}$ (characteristic peak of azide group), again confirmed the excess $\mathrm{PEO}_{23}-\mathrm{N}_{3}$ was completely removed. The UV-Vis curve of the purified T-shaped polymer (Fig. 3A, olive) shows a similar pattern as the crude product. Fig. 5A shows the ${ }^{1} \mathrm{H}$ NMR spectrum of the T-shaped polymer. The peak area ratio of $\mathrm{H}_{b}\left(170 \mathrm{H}\right.$ of PS) and $\mathrm{H}_{\mathrm{c}}(2 \mathrm{H})$ was approximately 85 , which was close to that of $\mathrm{H}_{\mathrm{h}}$ and $\mathrm{H}_{\mathrm{k}}\left(\mathrm{Area}_{\mathrm{Hh}} / \mathrm{Area}_{\mathrm{Hk}}=87\right)$ in Fig. 1 . It indicated the linear PS was completely consumed for the construction of T-shaped polymers. It is notable that the peak area of $\mathrm{H}_{\mathrm{a}}$ (82.56) was slightly smaller than the theoretical value (94), indicating a shorter PEO chain connected onto linear PS. It is becuase the shorter PEO chians had relatively higher reacivity than the longer ones, due to the smaller steric effects.
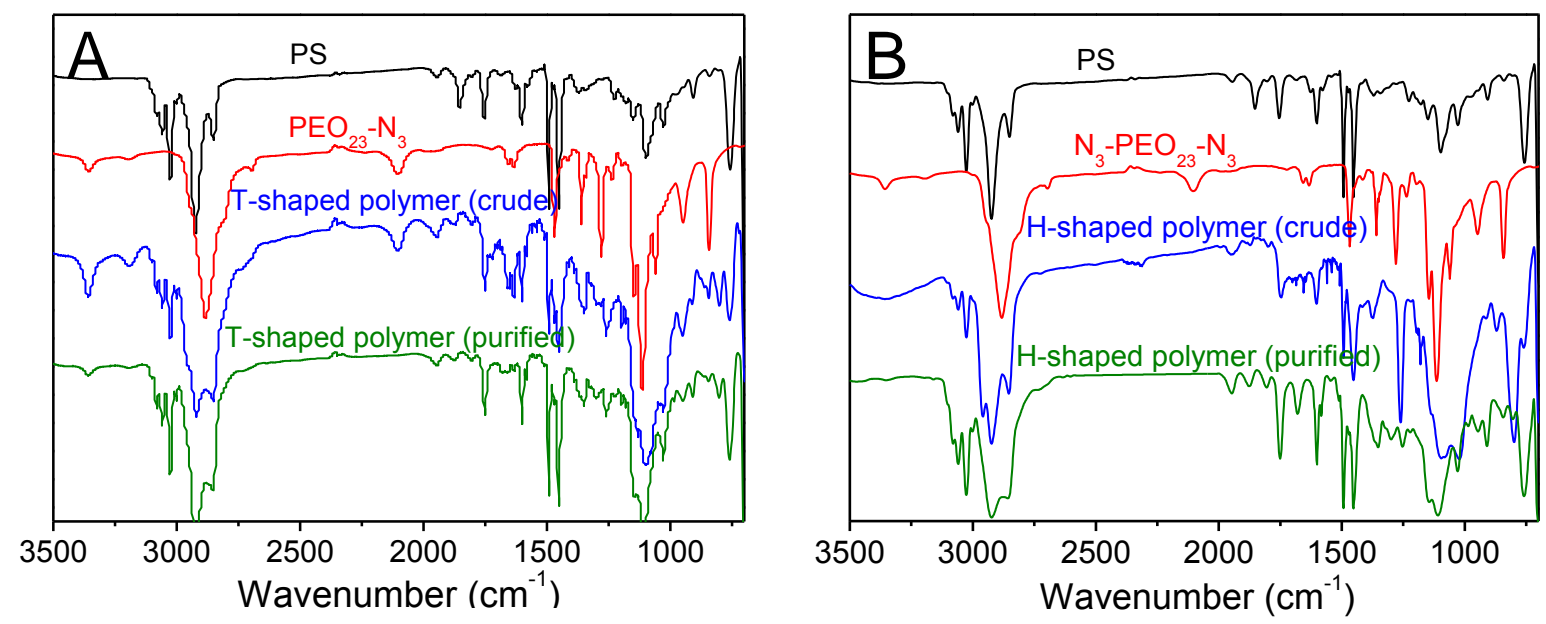

Fig. 4. (A) IR spectra of PS (black), $\mathrm{PEO}_{23}-\mathrm{N}_{3}$ (red), crude (blue) and purified (olive) T-shaped polymers. (B) IR spectra of PS (black), $\mathrm{N}_{3}-\mathrm{PEO}_{23}-\mathrm{N}_{3}$ (red), crude (blue) and purified (olive) $\mathrm{H}$-shaped polymers. 

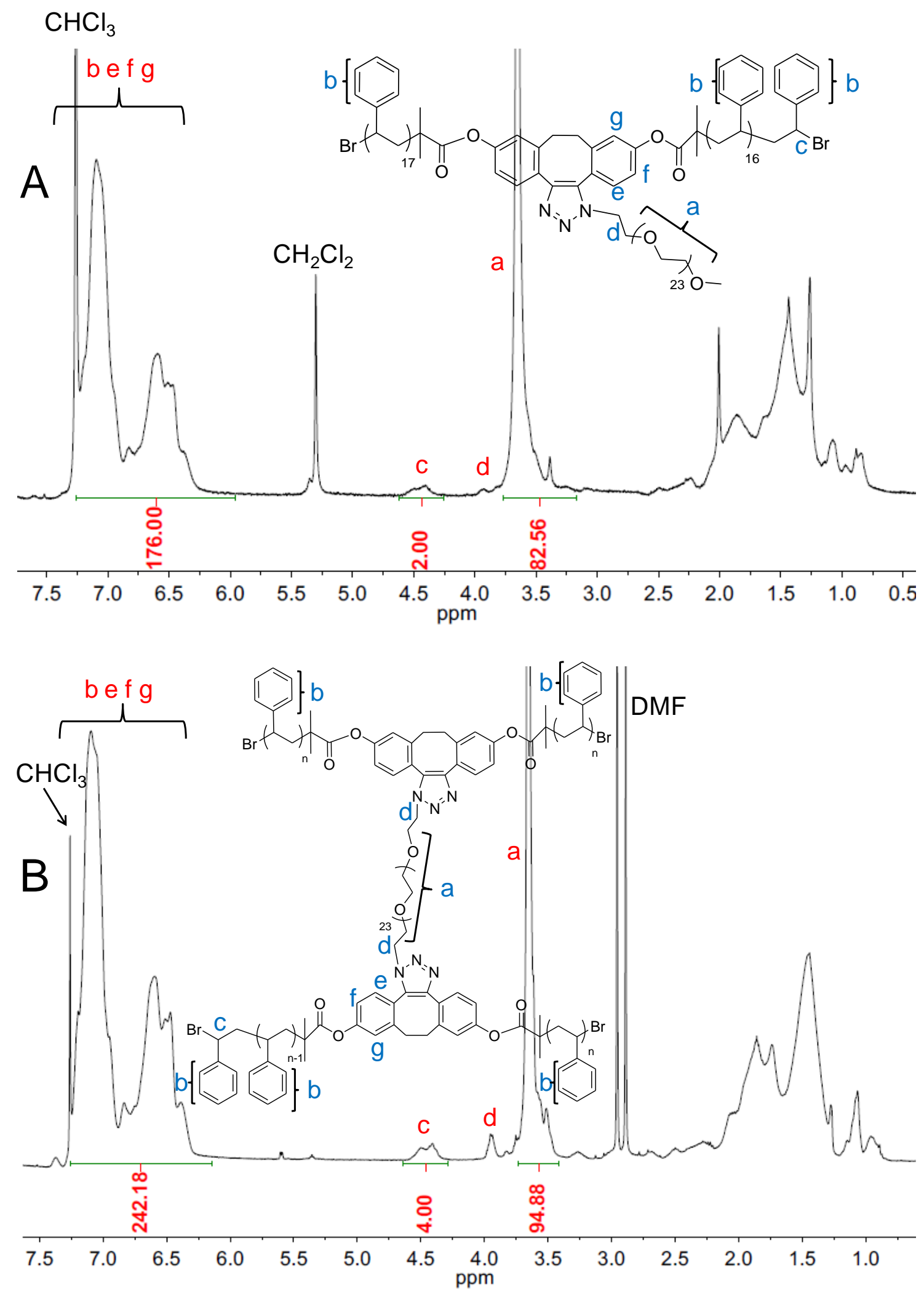

Fig. 5. ${ }^{1} \mathrm{H}$ NMR spectra of (A) T-shaped and (B) $\mathrm{H}$-shaped polymers in $\mathrm{CDCl}_{3}$. 


\subsection{Synthesis and characterization of $H$-shaped polymer}

$\mathrm{H}$-shaped polymer was composed of two molecules of linear PS linked by a PEO chain via the UV-induced SPAAC. The PEO chain, $\mathrm{N}_{3}-\mathrm{PEO}_{23}-\mathrm{N}_{3}$, has azide groups on both ends, which was synthesized according to the route of Fig. S4. Fig. S6 shows the ${ }^{1} \mathrm{H}$ NMR spectrum of $\mathrm{N}_{3}-\mathrm{PEO}_{23}-\mathrm{N}_{3}$. According to the peak area ratio of $\mathrm{H}_{\mathrm{a}}(96 \mathrm{H})$ and $\mathrm{H}_{\mathrm{b}}(4 \mathrm{H})$, the hydroxy groups on both ends of PEO had completely transformed to azide groups. The $M_{\mathrm{n}, \mathrm{NMR}}$ of $\mathrm{N}_{3}-\mathrm{PEO}_{23}-\mathrm{N}_{3}$ was 1170 . Fig. $4 \mathrm{~B}$ (red) shows the IR spectrum of $\mathrm{N}_{3}-\mathrm{PEO}_{23}-\mathrm{N}_{3}$, and the characteristic peak at $2100 \mathrm{~cm}^{-1}$ again confirmed the successful introduction of azide groups. Fig. 2B (red) shows the GPC curve of $\mathrm{N}_{3}-\mathrm{PEO}_{23}-\mathrm{N}_{3}$, and the $M_{\mathrm{n}, \mathrm{GPC}}$ and $M_{\mathrm{w}} / M_{\mathrm{n}}$ were integrated as 2100 and 1.03 , respectively.

The UV irradiation condition was the same as above to deprotect the cyclopropenone-masked dibenzocyclooctyne group. To ensure the product was H-shaped polymer, 0.5 equivalent (azide/ dibenzocyclooctyne $=1 / 2$ ) of $\mathrm{N}_{3}-\mathrm{PEO}_{23}-\mathrm{N}_{3}$ was added to the dibenzocyclooctyne functionalized PS solution. After $36 \mathrm{~h}$ reaction at room temperature in air, H-shaped PS-PEO copolymer was obtained. The crude product was characterized by GPC, UV-Vis and IR. The GPC curve (Fig. 2B, blue) of the crude product of $\mathrm{H}$-shaped polymer shows no peak of $\mathrm{N}_{3}-\mathrm{PEO}_{23}-\mathrm{N}_{3}$, but a peak of residual PS. The newly generated peak was moved to a higher molecular weight direction, but remained the mono-modal and symmetric peak shape compared to that of PS (black). This clearly indicated the successful preparation of the H-shaped polymer via the UV-induced SPAAC reaction. The IR spectrum of the crude product (Fig. 4B, blue) shows no characteristic peak of azide group at $2100 \mathrm{~cm}^{-1}$, indicating $\mathrm{N}_{3}-\mathrm{PEO}_{23}-\mathrm{N}_{3}$ was completely consumed. In the UV-Vis spectrum of Fig. 3B (blue), a small peak at $314 \mathrm{~nm}$ was observed, 
indicating a residual of dibenzocyclooctyne functionalized PS.

The crude product was purified by GPC fractionation to remove excess PS. GPC curve of the purified H-shaped polymer was shown in Fig. 2B (olive), in which a trace amount of unreacted PS could not be removed completely although it slightly affected the related analysis. The $M_{\mathrm{n}, \mathrm{GPC}}$ and $M_{\mathrm{w}} / M_{\mathrm{n}}$ were integrated as 9600 and 1.02, respectively. The UV-Vis curve of the purified H-shaped polymer was shown in Fig. 3B (olive), in which the dibenzocyclooctyne absorption peak was hardly observed at 314 nm. Fig. 5B shows the ${ }^{1} \mathrm{H}$ NMR spectrum of the $\mathrm{H}$-shaped polymer. The peak area ratio of $\mathrm{H}_{\mathrm{a}}(96 \mathrm{H}$ of PEO) and $\mathrm{H}_{\mathrm{c}}(4 \mathrm{H})$ was 23.7 , which was close to the theoretical value of 24 . However, the peak area (241.28) of $\mathrm{H}_{\mathrm{b}}$ was significantly less than its theoretical value (340), indicating shorter PS chains connected in the H-shaped polymers. It is becuase the shorter PS chians had relatively higher reacivity than the longer ones, due to the smaller steric effects. As shown in Fig. 2B (blue curve), the residual PS possessed a shorter elution time than the reactant PS (black curve), which indicated that the residual PS did have a larger $M_{\mathrm{n}, \mathrm{GPC}}$ and the shorter PS chains were consumed preferentially.

\section{Conclusions}

UV-induced SPAAC reaction has been demonstrated as a powerful tool for the construction of topological polymers, since the reaction between cyclopropenone-masked dibenzocyclooctyne and azide could be carried out under mild conditions without any catalyst but UV irradiation. Using a cyclopropenone-masked dibenzocyclooctyne functionalized dibromo ATRP initiator, linear PS was synthesized with a cyclopropenone-masked dibenzocyclooctyne in the middle of polymer chain. Well-defined T-shaped and H-shaped polymers were then produced by coupling PS with mono- and 
di-end azide functionalized linear PEO, respectively. Due to its clean and efficient reaction characteristics, UV-induced SPAAC reaction is a golden candidate for preparing varied topological polymers required in photoelectronic materials, biomedical materials, and many other related applications.

\section{Acknowledgments}

Generous supports were primarily provided by Ministry of Science and Technology of China (2014CB932200), National Science Foundation of China (21374122 and 51203171), Beijing National Laboratory for Molecular Sciences, and Bairen project from The Chinese Academy of Sciences.

\section{References}

[1] Tezuka Y, Oike H. Prog Polym Sci 2002;27:1069-122.

[2] Ivan B. Macromol Chem Phys 2000;201:2621-8.

[3] Gao H, Matyjaszewski K. Macromolecules 2006;39:4960-5.

[4] Gao H, Matyjaszewski K. J Am Chem Soc 2007;129:6633-9.

[5] Tsarevsky NV, Bencherif SA, Matyjaszewski K. Macromolecules 2007;40:4439-45.

[6] Konkolewicz D, Gray-Weale A, Perrier S. J Am Chem Soc 2009;131:18075-7.

[7] Eugene DM, Grayson SM. Macromolecules 2008;41:5082-4.

[8] Binder WH, Petraru L, Roth T, Groh PW, Palfi V, Keki S, Ivan B. Adv Funct Mater 2007;17: $1317-26$.

[9] Zhang YF, Li CH, Liu SY. J Polym Sci Pol Chem 2009;47:3066-77. 
[10] Altintas O, Hizal G, Tunca U. J Polym Sci Pol Chem 2006;44:5699-707.

[11]Yuan WZ, Zhang JC, Zou H, Ren J. J Polym Sci Pol Chem 2012;50:2541-52.

[12]Mu CG, Fan XD, Tian W, Bai Y, Yang Z, Fan WW, Chen H. Polym Chem 2012;3:3330-9.

[13]Li LY, He WD, Li WT, Zhang KR, Pan TT, Ding ZL, Zhang BY. J Polym Sci Pol Chem 2010;48:5018-29.

[14]Dag A, Durmaz H, Hizal G, Tunca U. J Polym Sci Pol Chem 2008;46:302-13.

[15]Durmaz H, Karatas F, Tunca U, Hizal G. J Polym Sci Pol Chem 2006;44:3947-57.

[16] Iskin B, Yilmaz G, Yagci Y. J Polym Sci Pol Chem 2011;49:2417-22.

[17]Chan JW, Yu B, Hoyle CE, Lowe AB. Chem Commun 2008;133:4959-61.

[18]Lutz JF. Angew Chem Int Ed 2008;47:2182-4.

[19] Agard NJ, Prescher JA, Bertozzi CR. J Am Chem Soc 2004;126:15046-7.

[20] Orski SV, Sheppard GR, Arumugam S, Arnold RM, Popik VV, Locklin J. Langmuir 2012;28:14693-702.

[21] Johnson JA, Baskin JM, Bertozzi CR, Koberstein JT, Turro NJ. Chem Commun 2008;133:3064-6.

[22] Yang XK, Wang SS, Yan YC, Wu Y, Zhang K, Chen YM. Polymer 2014;55:1128-35.

[23] Wang SS, Yang XK, Zhu W, Zou L, Zhang K, Chen YM, Xi F. Polymer 2014;55:4812-9.

[24] Sun P, Yan GW, Tang QQ, Chen YM, Zhang K. Polymer 2015;64:202-9.

[25] Sun P, Tang QQ, Wang ZP, Zhao YM, Zhang K. Polym Chem 2015;6:4096-101.

[26] Tang QQ, Chen JQ, Zhao Y, Zhang K. Polym Chem 2015;6:6659-63. 


\section{Graphical Abstract}

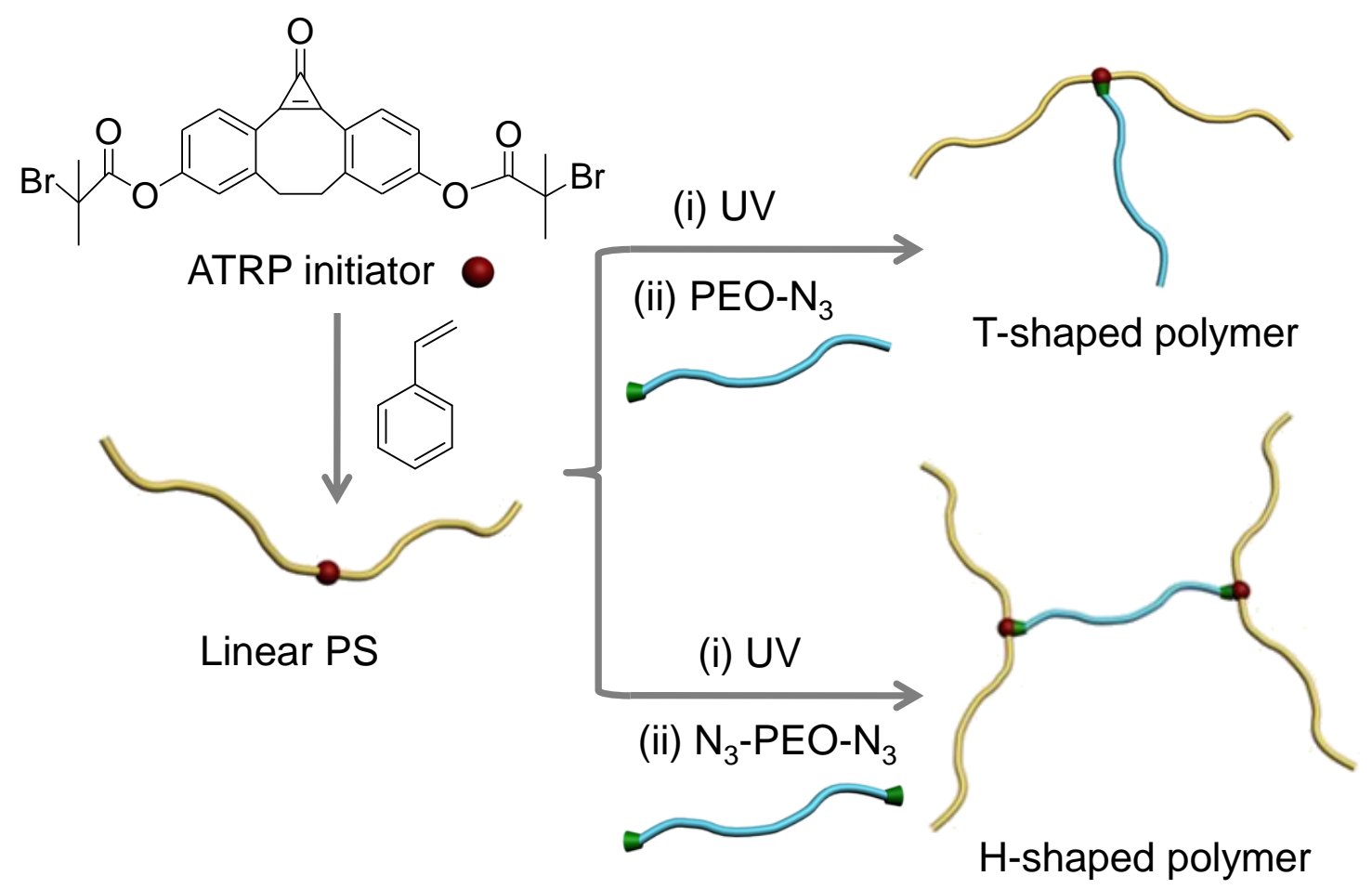

